\title{
Standardization of First and Second-line Antitubercular Susceptibility Testing Using BacT Alert 3D System- A Report from a Tertiary Care Centre in India
}

\author{
Deepthi Nair ${ }^{1}$, Malini R. Capoor ${ }^{2}$, Deepti Rawat ${ }^{3}$, Lakshmi Srivastava ${ }^{4}$ and Pushpa Aggarwal ${ }^{5}$ \\ ${ }^{1}$ Senior Specialist \& Assistant Professor, ${ }^{2}$ Specialist, ${ }^{3}$ Senior Research Fellow, ${ }^{4}$ Consultant, ${ }^{5}$ Consultant \& Head of Department \\ Department of Medical Microbiology, Safdarjung Hospital \& Vardhman Mahaveer Medical College; New Delhi, India
}

\begin{abstract}
Resurgence of multidrug resistant tuberculosis has lead to demand for rapid susceptibility testing. Conventional methods take $>3$ weeks and are tedious. Automated methods have superseded them for first line drug susceptibility testing. An attempt was made to standardize first and second line susceptibility testing using the BacT Alert 3D system (Biomerieux). And compare results with Lowenstein Jensen's (LJ) method. 121 isolates of Mycobacterium tuberculosis, 67 pulmonary and 54 extra pulmonary were subjected to sensitivity to first and second line drugs. Multidrug resistance was detected equally by both methods at $15.7 \% .100 \%$ agreement was observed between the two methods for aminoglycosides, rifampicin, ethionamide and ciprofloxacin. $\mathbf{9 1 . 5 \%}$ agreement was observed for isoniazid, $85 \%$ for pyrazinamide and $72.4 \%$ for ethambutol. The time taken by LJ method was 18-32 days and BacT Alert 3D system took 4-12 days. In the lesser developed nations where tuberculosis is rampant a rapid effective method for confirming multidrug resistant tuberculosis is definitely desirable and the BacT Alert 3D system was found an effective method when compared to the 'gold standard' LJ proportion.
\end{abstract}

Key-Words: Antitubercular sensitivity, second line, BacT Alert 3D system, LJ proportion.

Mycobacterium tuberculosis is one of the most successful of human pathogen. About a third of the world's population is infected with it. There are approximately 9 million new cases of every year, with 2 million deaths [1]. The global resurgence of tuberculosis (TB) and the increase in multidrug resistant (MDRTB) strains has led to a concomitant increase in demand of susceptibility testing of isolates to first (Streptomycin, Isoniazid, Rifampicin, Ethambutol, Pyrazinamide) and secondline antitubercular (ATT) drugs (Ethionamide, Amikacin, Kanamycin, Capreomycin, Ciprofloxacin, Ofloxacin, Levofloxacin, Cycloserine, Rifabutin etc). Using the conventional antimicrobial susceptibility testing (AST) with solid media such as Lowenstein-Jensen (LJ) proportion or Middle brook 7H 10 or 11 agar, time taken is usually more than 3 weeks and the procedure is tedious [2].

Drug resistance in tuberculosis has been prevalent from the time the drugs were introduced for therapy. In India, most information of the drug resistance is based on presumptive clinical diagnosis as facilities for sputum culture and susceptibility for mycobacteria are restricted to limited centers [3]. In 1993, the Centers for Disease Control and prevention (CDC) laid down the criteria for the diagnosis of $M$. tuberculosis [4]. The results of smear should be available within 24 hours of collection, culture in liquid medium should be done to detect growth in 10-14 days and AST should be available in 15-30 days after collection of sample. As a direct corollary to this there was an increase in use of automation in mycobacterial culture and sensitivity. The first system used for detection of Mycobacterium in clinical laboratories was the radiometric, semi-automated BACTEC 460. This had

Received on 6 May 2009; revised 13 November 2009.

Address for correspondence: Dr. Deepthi Nair. D-II/2201, Vasant Kunj. New Delhi-110070, India. E-mail: deepthinair2@gmail.com.

The Brazilian Journal of Infectious Diseases 2009;13(6):422-426. (C) 2009 by The Brazilian Journal of Infectious Diseases and Contexto Publishing. All rights reserved. several drawbacks like radioactivity, increased exposure to the infection due to manual handling, invasive technique and increased rates of cross-contamination [5]. Subsequently, several systems were developed which used non-radiometric methods of estimating growth. The foremost amongst these are the BacT Alert 3D system, ESP/Trek Systems, Mycobacterial Growth Indicator Tube 960 (MGIT), etc. [6-8]. The radiometric and non-radiometric systems have been evaluated extensively for isolation and susceptibility to first line anti tubercular agents $[9,10,11,12,13,14]$. Very few studies have been conducted to evaluate the second line antitubercular drug susceptibility testing $[2,14,15]$.

In the current study an attempt was made to standardize first and second line anti tubercular drug susceptibility testing using the BacT Alert 3D system in a DOTS referral centre, in a tertiary care government- run hospital in New Delhi, India.

\section{Material and Methods}

Study Site and Strains

A total of 13,982 samples were received in the laboratory over a 35-month period (August 2002-June 2005). Amongst these $11,468(78.5 \%)$ were pulmonary and 2514(17.2\%) extra pulmonary (synovial aspirate, lymph node aspirate, tissue biopsy, CSF, pus from abscess etc) cases. All samples were processed as described below and stained by Ziehl Neelson's method. Pulmonary samples (2371) from patients who showed recurrence or therapeutic failure (Cat II of DOTS) were processed by culture using manual (LJ method) and automation (BacT/ Alert 3D, Biomerieux, France). All (2514) extra pulmonary samples were processed by both culture methods.

An average recovery rate of $61.9 \%$ was observed in pulmonary samples and 33\% in extra pulmonary isolates.121 isolates of Mycobacterium tuberculosis were randomly selected, 67 pulmonary and 54 extra pulmonary strains were subjected to first and second line susceptibility testing using both methods. 
Samples Processing for Culture

\section{LJ Method [16]}

Decontamination of the samples was performed using the modified Petroff's method. The deposit was inoculated onto $\mathrm{LJ}$ medium and incubated at $35-37^{\circ} \mathrm{C}$ aerobically. The samples from sterile sites were inoculated without decontaminationsterile clear fluids were centrifuged and the deposit was inoculated, turbid thick pus was inoculated directly. The LJ cultures were observed bi-weekly till a period of 8 weeks.

\section{Automation; BacT Alert 3D System, [5,6]}

The BacT/Alert MP contains the modified Middle brook 7H9 broth with casein, bovine albumen and catalase. To 10ml of this medium, $0.5 \mathrm{ml}$ of reconstitution fluid (Tween 80 , glycerol and amaranth) was added, with or without antibiotics, depending upon whether the sample was contaminated. The antibiotics supplement (MAS supplement) consisted of trimethoprim and vancomycin. Samples were decontaminated and $0.5 \mathrm{ml}$ of the processed sample was inoculated aseptically into the BacT/Alert MP bottle. These were then inserted for incubation and detection in the equipment. The bottles were read every ten minutes in the machine and as a bottle flagged positive, it was removed and further identified.

\section{Confirmation of Isolates}

All positive cultures were confirmed using various conventional tests as described [17]. These included ZN smear, catalase, nitrate reduction test, para nitro benzoic acid testing and Accuprobe test for confirmation of $M$. tuberculosis complex [18].

Antimicrobial Susceptibility Testing

AST was performed by the conventional proportion method using $\mathrm{LJ}$ medium and automation in the BacT Alert 3D system[6,9,10,11,12,13,14,15,16]. All the LJ subcultures of Mycobacteria were $\leq 3$ weeks old and $\leq 36$ hours, in the BacT/ Alert MP bottle.

\section{Antimicrobial Agents}

The drug powders were obtained from Sigma, St Louis MO. The first line agents were: streptomycin, isonizaid, rifampcin, ethambutol, pyrazinamide. The second line agents tested were: ciprofloxacin, ofloxacin, amikacin, capreomycin, kanamycin and ethionamide. All the agents were dissolved in sterile distilled water (SDW) to make the master solution $(10,000 \mu \mathrm{g} / \mathrm{mL})$. The stock solutions were stored in small aliquots at $-70^{\circ} \mathrm{C}$. Working solution $(1000 \mu \mathrm{g} / \mathrm{mL})$ was prepared on the day of the tests and added to the LJ medium and MB Bact medium to achieve the critical concentration (Table 1) [2,6,9,11-15]. At least 3 critical concentration of each of the drugs were tested in the BacT Alert 3D system to determine the ideal concentration for the strains prevailing in the local environment Pfyffer et al. 1999). For proportion testing using LJ medium only relevant critical concentrations were used [16].
Table 1. Critical concentration (ug/mL) for $1 \%$ proportion testing of antimicrobial agents used. [6,9-16].

\begin{tabular}{lcc}
\hline & MB Bact bottles LJ medium \\
\hline First line & & \\
Streptomycin & $1,2,4$ & 4 \\
Isonizaid & $0.1,0.2,1$ & 0.2 \\
Rifampcin & 1,2 & 40 \\
Ethambutol & $2,2.5,5,7.5$ & 2 \\
Pyrazinamide & 25,100 & 100 \\
Second line & & \\
Capreomycin & $1.25,2.5,5$ & 40 \\
Kanamycin & $1.25,2.5,5$ & 30 \\
Amikacin & $2.5,5$ & 30 \\
Ethionamide & $1.25,2.5,5$ & 30 \\
Ciprofloxacin & $2,4,6,8$ & 2 \\
Ofloxacin & $2,4,6,8$ & 2 \\
\hline
\end{tabular}

LJ Proportion Method [16]

The bacterial suspension was prepared by picking 2-3 loopful (3mm internal diameter) of colonies into $0.2 \mathrm{ml}$ sterile distilled water in a screw capped (approximately 4mg moist weight of the growth on LJ medium). The colonies on the drug free medium expressed as a percentage. One percent or more of growth is considered as resistant for all drugs.

\section{BacT Alert 3D Proportion Method [6,9-15]}

The BacT/Alert MP bottle containing the growth of Mycobacterium tuberculosis ( $\leq 36$ hours, subculture, then the growth was diluted 1:1 in sterile distilled water). This formed the direct growth control of approximately Mc Farland no.2 (DGC). This was put into all drug containing and control bottles. A 100 -fold dilution of the DGC $(0.1 \mathrm{ml}$ of DGC $+9.9 \mathrm{ml}$ of SDW) was prepared and $0.5 \mathrm{ml}$ was added to another BacT/ Alert MP bottle and this was the $1 \%$ growth control (1\% GC). These bottles were incubated in the system at $35^{\circ} \mathrm{C}$ for 12 days and monitored every $10 \mathrm{~min}$ to detect growth.

An isolate was considered as resistant if the bottles containing the drug flagged positive at the same time or before the 1\% GC. An isolate was considered susceptible if the bottle containing the drug remained negative during the test period or flagged positive after the 1\% GC. If the DGC did not flag positive in 12 days the test was invalidated and had to be repeated.

\section{Results}

The resistance pattern of the drugs, as detected by LJ which was taken as the 'gold standard' for comparison, was in the order of isoniazid being the least effective (44.6\%) followed by ethambutol (17.4\%) and rifampcin (Table 2). Monodrug resistance was found in 26(21.5\%) isolates,

Isoniazid was the least effective single agent. Resistance to 2 drugs was observed predominantly with INH and rifampicin (15.7\%), 3 drug resistance was observed to INH, rifampicin and 
Table 2. Resistance pattern towards first and second line drugs by LJ proportion method versus BacT Alert method.

\begin{tabular}{lcccccccccc}
\hline Antibiotics & $\mathbf{S}$ & $\mathbf{I}$ & $\mathbf{R}$ & $\mathbf{E}$ & $\mathbf{P y z}$ & $\mathbf{E t h i o}$ & $\mathbf{C i p}$ & $\mathbf{A k}$ & $\mathbf{K}$ & Capr \\
\hline Pulm & & & & & & & & & & \\
$\quad$ LJ & $8 / 67$ & $38 / 67$ & $13 / 67$ & $17 / 67$ & $10 / 67$ & $6 / 57$ & $2 / 57$ & $3 / 57$ & $3 / 57$ & $3 / 57$ \\
$\quad$ BacT & $8 / 67$ & $41 / 67$ & $13 / 67$ & $19 / 67$ & $13 / 67$ & $16 / 67$ & $2 / 67$ & $5 / 67$ & $3 / 67$ & $6 / 67$ \\
Extra pulm & & & & & & & & & & \\
$\quad$ L & $3 / 54$ & $16 / 54$ & $8 / 54$ & $4 / 54$ & $7 / 54$ & $6 / 54$ & $5 / 54$ & $5 / 54$ & $6 / 54$ & $6 / 54$ \\
$\quad$ BacT & $3 / 54$ & $18 / 54$ & $8 / 54$ & $10 / 54$ & $7 / 54$ & $8 / 54$ & $2 / 54$ & $3 / 54$ & $2 / 54$ & $2 / 54$ \\
Total & & & & & & & & & & \\
$\quad$ LJ & $11 / 121$ & $54 / 121$ & $21 / 121$ & $21 / 121$ & $17 / 121$ & $14 / 121$ & $7 / 121$ & $8 / 121$ & $9 / 121$ & $9 / 121$ \\
& $(9.1)$ & & $(17.4)$ & $(17.4)$ & $(14.1)$ & $(11.6)$ & $(5.8)$ & $(6.6)$ & $(7.4)$ & $(7.4)$ \\
$\quad$ BacT & $11 / 121$ & $59 / 121$ & $21 / 121$ & $29 / 121$ & $20 / 121$ & $24 / 121$ & $4 / 121$ & $8 / 121$ & $5 / 121$ & $8 / 121$ \\
& $(9.1)$ & $(48.8)$ & $(17.4)$ & $(17.4)$ & $(16.5)$ & $(23.9)$ & $(3.3)$ & $(6.6)$ & $(4.1)$ & $(6.6)$ \\
\hline
\end{tabular}

S-Streptomycin, Ethio- Ethionamide, I-Isoniazid, Cip-Ciprofloxacin, R- Rifampcin, Ak-Amikacin, E- Ethambutol, K-Kanamycin, Pyz-Pyrazinamide, Capr-Capreomycin.

Table 3. Drug resistance in pulmonary and extra pulmonary isolates.

\begin{tabular}{lccc}
\hline & Pulmonary & $\begin{array}{c}\text { Extra } \\
\text { pulmonary }\end{array}$ & Total \\
\hline Single drug resistance & $18 / 16$ & $8 / 6$ & $26 / 22$ \\
Two drug resistance & $13 / 13$ & $4 / 4$ & $17 / 17$ \\
Three drug resistance & $8 / 7$ & $3 / 3$ & $11 / 10$ \\
$>$ 3 drug resistance & $5 / 3$ & $1 / 1$ & $6 / 4$ \\
All resistant & $2 / 2$ & $2 / 2$ & $4 / 4$ \\
All sensitive & $21 / 21$ & $36 / 34$ & $57 / 55$ \\
Total & 67 & 54 & 121 \\
\hline
\end{tabular}

Most common pattern: Monodrug: I = Pulmonary $13(10.7)+$ Extra pulmonary 6 (4.96) $=19$ (14.9); 2 drug: $\mathrm{I}+\mathrm{R}=$ Pulmonary 5 $(4.13)+$ Extra pulmonary 2 (1.65) $=7$ (5.8); I + E = Pulmonary 2 $(1.65)+$ Extra pulmonary $1(0.08)=3(2.48)$; $>3$ drug: $\mathrm{I}+\mathrm{R}+\mathrm{E}=$ 7 (5.8). Mono rifampicin resistance observed in 2 isolates (1.7\%).

Thus total 21 (17.4\%) resistance observed to rifampicin.

ethambutol, resistance to $>3$ drugs was seen in $8.3 \%$ cases (Table 3). There was no mono-drug resistance seen to Streptomycin, Capreomycin, Amikacin, Ciprofloxacin and Ofloxacin. Fifty seven strains (47.1\%) were sensitive to all drugs tested. Resistance to all the drugs was observed in 2 isolates (1.7\%) each of pulmonary and extra pulmonary cases (Table 3 ).

Multidrug resistant isolates (Isoniazid and Rifampicin) were $15.7 \%$ by both the methods (Tables 2 and 3) MDRTB was 1.7 times more in pulmonary isolates as compared to extra pulmonary using both methods. Amongst the second line drugs maximum resistance was seen to Kanamycin (7.4\%) (Tables 2 and 3) There were 4 strains that were resistant to quinolones and these were resistant to all the other drugs tested in the study. Two of the isolates were recovered from two patients who had extra pulmonary TB (synovial tuberculosis and jaw abscess) which went on to become miliary tuberculosis as both the patients were immune-compromised. One of the patients with an all resistant isolate had pulmonary disease was lost to follow up and the sputum of the fourth patient continued to be positive for Mycobacterium tuberculosis in spite of medication and eventually the patient expired.
Table 4. Correlation between resistance results of BacT Alert vs. LJ proportion method for first and second-line antitubercular agents.

\begin{tabular}{lccc}
\hline Drug N=121 & Bac T Alert & LJ & Correlation \\
\hline Streptomycin & 11 & 11 & $100 \%$ \\
Isoniazid & 59 & 54 & $91.5 \%$ \\
Rifampcin & 21 & 21 & $100 \%$ \\
Ethambutol & 29 & 21 & $72.4 \%$ \\
Pyrazinamide & 20 & 17 & $85 \%$ \\
Ethionamide & 8 & 8 & $100 \%$ \\
Ciprofloxacin & 4 & 4 & $100 \%$ \\
Amikacin & 5 & 5 & $100 \%$ \\
Kanamycin & 5 & 5 & $100 \%$ \\
Capreomycin & 5 & 5 & $100 \%$ \\
\hline
\end{tabular}

The comparison (percentage agreement) between LJ and Bact Alert $3 \mathrm{D}$ proportion method toward $1^{\text {st }}$ and $2^{\text {nd }}$ line antitubercular drugs is shown in Table $4.100 \%$ agreement was observed for Streptomycin, Rifampicin, Ethionamide, Ciprofloxacin, Amikacin, Capreomycin and Kanamycin. There was $91.5 \%$ agreement for Isonizaid, $85 \%$ for pyrazinamide and $72.4 \%$ for Ethambutol (Table 4). As recommended by the IUATLD (2001) sensitivity, specificity, positive predictive value (PPV) and negative predictive value (NPV) of each antimicrobial for the colorimetric system were determined as compared with LJ method. The sensitivity was 100\% for all the first and second-line antimicrobials. Specificity was $100 \%$ for Streptomycin, Rifampicin, Ethionamide, Ciprofloxacin, Ofloxacin, Amikacin, Kanamyan and Capreomycin. Specificity was 92.6\% for Ethambutol, 93.1\% for Isoniazid and 97.2\% for Pyrazinamide. Positive predictive value was $100 \%$ for Streptomycin, Rifampicin, Ethionamide, Ciprofloxacin, Ofloxacin, Amikacin, Kanamycin and Capreomycin. It was lower for Ethambutol, 68\%, 87\% Pyrazinamide and 91.5\% for Isoniazid. The negative predictive value was $100 \%$ for all the drugs. 
The average time taken to detect resistance by the $\mathrm{LJ}$ proportion method ranged from 18-32 days with an average of 20 days. The BacT ALERT 3D system took 4-12 days with an average of 8 days. There was a $40 \%$ reduction in time using the automated machine. The drug resistant isolates took $\geq 10$ 12 days by automation and 27-32 days by LJ method to grow which, was longer than the sensitive isolates.

\section{Discussion}

In India 8.8 million persons are infected with tuberculosis and 3.5 million are open cases. Every year there are 2.2 million fresh cases [16].

There are several problems compounding the issue. The parallel Hiv epidemic, socio-economic constraints, primary and secondary MDR TB and diagnostic delays are factors contributing to spread of this disease [15]. The basis of the RNTCP, India, which follow the DOTS guidelines, is smear microscopy. This is a cheap test although it has low specificity and variable sensitivity [16]. Conventional methods of isolation although specific, take 4-6 weeks time and have variable sensitivity depending on the type of sample. Susceptibility tests take another 3-4 weeks time[2,4]. This delay can result in increased transmission of tuberculosis, which is hazardous in cases of MDR TB. Thus rapid diagnosis and results of sensitivity are crucial in curtailing this emerging disease. Automation and molecular biology have contributed significantly in TB diagnostics.

The radiometric BACTEC 460 system was the earliest method of automation to be tried and antimicrobial susceptibility testing by this method is considered to be the gold standard in automation. It is a rapid and effective method but the use of radioisotopes and semi automated technique are a definite disadvantage [5]. There are several nonradiometric techniques, these include the colorimetric BacT ALERT 3D system, MGIT, ESP-II /TREK system, phage based assays, Micro well Alamar blue assays, Bioluminescence method, MODS etc. [6,8,9,19-21].

The colorimetric method using the BacT Alert 3D system is relatively new. There are reports of antibiotic sensitivity testing using this method, especially for second line antitubercular agents [2,6,11,22-24]. This system could perform the susceptibility testing in 8 days compared to 20 days by the LJ-method. There was a $40 \%$ reduction in time using the automated machine. The drug resistant isolates took $\geq 10$ - 12 days by automation and $27-32$ days by LJ method to grow which, was longer than the sensitive isolates. The drug resistant isolates took longer to grow compared to the sensitive strains by automation; a similar finding has been reported by Toungoussova et al. [24]. The time taken to perform susceptibility was well within the time frame of 4weeks delineated by Tenover et al. [25].

The critical concentration for use with the BacT ALERT system has been elucidated earliest by Beer et al. and Pfyffer et al. using the BACTEC $[2,6]$. In the current study three to four different concentrations were tested for each drug and each test was repeated three times to validate results [2].This was then compared with the LJ proportion method. There was
$100 \%$ agreement in drug susceptibility testing by the two methods for rifampicin, streptomycin, ethionamide, ciprofloxacin, ofloxacin, amikacin, capreomycin and kanamycin. Similar results were observed by several workers ranging from $90-100 \%$ for various drug tested [2,9-15,22-24].

The sensitivity to detect resistance was high for all drugs at $100 \%$. The specificity of automated system i.e. its capacity to detect sensitive strains was also high at $100 \%$ for streptomycin, rifampicin, ethionamide, ciprofloxacin, ofloxacin, amikacin, kanamycin and capreomycin. In present study lower sensitivity was observed for pyrazinamide (98.3\%), isoniazid (95\%) and ethambutol (94.7\%). 100\% sensitivity and specificity was reported for Rifampcin, followed by INH and pyrazinamide although streptomycin and ethambutol gave lower sensitivity and specificity [12]. Angeby KAK et al. have reported 100\% specificity to first line drugs and $100 \%$ sensitivity for ethambutol followed by $96 \%$ for isonizaid, 92\% for rifampcin and 78\% for streptomycin [10]. Barreto et al. reported 100\% sensitivity and specificity of or amikacin, rifampicin and ofloxacin [11,14].

Poor correlation with false resistance by BacT Alert 3D system was observed to ethambutol and isonizaid; such inaccuracies have been reported for ethambutol by other workers $[12,14]$. Ethambutol testing using the BacT Alert 3D system has been a subject of review due to certain factors like presence of borderline resistant isolates which are picked up better by the solid media where accurate colony counting is possible. The low PPV with ethambutol (68\%) as seen by us was also observed by Brunello and Fontana (54.5\%). They explain this to be due to the drug being bacteriostatic and also the mechanism of detecting growth in the BacT Alert 3D, which detects $\mathrm{CO} 2$ production. Ethambutol being bacteriostatic, all the TB bacilli in the bottle are not killed, the borderline resistant strains that survive produce enough $\mathrm{CO} 2$ to cause a detection in growth sensors which gives rise to a positive flash and detection as resistant. This is particularly true if the initial inoculum is more than 0.5-1 MacFarland and the MIC of the strain is borderline i.e. near the critical concentration [12]. According to Madison et al. use of two critical concentration of Ethambutol (a low and high concentration) also did not give any further improvement in accuracy when compared to agar proportion [26]. This could be important as ethambutol is used in first line regimen. Another significant factor is the presence of two large cohorts of multi drug resistant isolates in India- the classical isoniazid and rifampicin and another to isoniazid and ethambutol, as observed in the current study.

MDRTB was detected in $15.7 \%$ isolates by both the methods in the study. This is very desirable that automation performed as well as conventional methods in detection of MDRTB. Similar levels of MDRTB have been reported by various workers from different parts of the country $16 \%$ by Hemrani et al. and a higher level was reported by Malhotra et al. (24.3\%) [27,28]. A recent report released by WHO suggests that primary drug resistance is $5 \%$ in India and secondary resistance is $17 \%$, which, is very similar to our results [1]. In some areas acquired MDRTB is as high as 58\% [29]. A recent 
study from North Pakistan reports 28\% MDRTB with INH having highest resistance at 37\% [30]. Isoniazid, mono-drug resistance was very high in the current study, $48.8 \%$ followed by ethambutol at $23.9 \%$.

Hemrani et al. have also reported high INH resistance at $65.2 \%$. They have also reported high resistance to second line agents, $28.9 \%$ to Kanamycin and $12 \%$ to ciprofloxacin. Globally also reports of increasing MDRTB have been documented over the years $[11,14,15]$.

In conclusion, automated methods of sensitivity testing could expedite laboratory results, greatly aiding clinicians in the management and control of drug resistant tuberculosis. The rapid methodology could be used to initiate automation at the peripheral health setting- a hitherto unmet need in countries like India.

\section{References}

1. Global tuberculosis control: surveillance, planning, financing. WHO World Health Organisation, Geneva, 2008. (WHO/HTM/TB/ 2008.393)

2. Pfyffer, G.E., Bonata, D.A., Ebrahimzadeh, A., Gross, W., Hotating, J., Kornblum, J.,Laszlo,A., Roberts, G., Salfinger, M., Wittmer, F., Siddiqi, S. Multicenter laboratory validation of susceptibility testing of Mycobacterium tuberculosis against classical second line and newer antimicrobial drugs by using the radiometric BACTEC 460 technique and the proportion method with solid media. J Clin Microbiol 1999;36: 3234-3238.

3. Revised National Tuberculosis Control Programme. DOTS Plus guidelines 2006; Central TB Division, Directorate general of health Services, Ministry of health and Family Welfare, Nirman Bhavan, New Delhi, India.

4. Centers for Disease Control and Prevention, 1997. Provisional cases of selected notifiable disease, United States, weeks ending Dec 27, 1997 and Dec 28, 1996 (52 ${ }^{\text {nd }}$ week). Morbid. Mortal. Weekly Rep. 46, 1261.

5. Rohner, P., Ninet,B., Mitral,C., Emler, S.,Auckenthaler, R. Evaluation of the MB/BacT system and comparison to the BACTEC 460 system and solid media for isolation of mycobacteria from clinical specimens. J. Clin. Microbiol 1997;35:3127-3131.

6. Beer J, Feldmann, K., Gogolin, J., Jaksch, G., Kuchler, R., Rifai, M., Rodloff, A.C., Rusch-Gerdes, S. (1997) Evaluation of the MB/ BacT drug susceptibility system for INH, RMP, SM, EMB. A cooperative study of 6 laboratories. Clin. Microbiol. Infect 1997;3.

7. Tortoli,E., Gichero,P., Chirillo,M.G. et al. Multicenter comparison of ESP culture system II with BACTEC 460 TB and with Lowenstein-Jensen Medium for recovery of mycobacteria from different clinical specimens, including blood. J Clin Microbiol 1998;36:1378-1381.

8. Cambau, E., Truffot-Pernot,C., Boulahbal,F., et al. (2000) Mycobacterial growth indicator tube versus the proportion method on Lowenstein Jensen medium for antibiotic susceptibility testing of Mycobacterium tuberculosis. Eur. J. Clin. Microbiol. Infect. Dis.19, 938-42.

9. Yew, W.W., Tong,S.C., Lui,K.S., Leung,S.K., Chau,C.H., EP Wang, E.P. Comparison of MB/BacT system and agar proportion method in drug susceptibility testing of Mycobacterium tuberculosis. Diag. Microbiol. Infect. Dis 2001;. 39: 229-32.

10. Angeby,K.A.K., Werngren,J., Toro,J.C., Hedstrom,G., Petrini,B., Hoffner,S.E. Evaluation of the BacT/ALERT 3D system for recovery and drug susceptibility testing of Mycobacterium tuberculosis. Clin. Microbiol. Infect.2003;91: 1148.

11. Barreto, A.M., Araujo, J.B., Medeiro,R.F., Caldas, P.C. Direct sensitivity test of the MB/BacT system. Mem. Inst. Oswaldo Cruz. 2002; 97:263-264.
12. Brunello, F. and Fontana, R. Reliability of the MB/BacT system for testing susceptibility of Mycobacterium tuberculosis complex isolates to antituberculous drugs. J. Clin. Microbiol. 2000;38:872-3.

13. Piersimoni,C., Scarparo,C., Callegaro,A., Tosi,C.P., Nisla,D., Bornigia,S., Scagnelli,M., Rigon,A., Ruggiero,G., Goglio,A. Comparison of MB/BacT ALERT 3D system with radiometric BACTEC system and Lowenstein-Jensen medium for recovery and identification of Mycobacteria from clinical specimens. A multicenter study. J. Clin. Microbiol.2001; 39: 651-657.

14. Barreto,A.M., Araiyo,J.B., Medeiro,R.F., Caldas,P.C. Evaluation of indirect susceptibility testing of Mycobacterium tuberculosis to the first and second-line and alternative drugs by the Newer MB/BacT system. Mem. Inst. Oswaldo Cruz. 2003;98:827-830.

15. Toungoussova,O.S., Mariandysher,A.O., Bjune,G., Caugant,D.A., Sandnen, I. Resistance of multidrug resistant strains of Mycobacterium tuberculosis from the Archangeloblast, Russia, to second-line anti-tuberculosis drugs. Eur. J. Clin. Microbiol. Infect. Dis.2005; 24:202-6.

16. Revised National Tuberculosis Control Programme.2007 Training Manual for Bacteriological aspects of Drug Resistance Surveillance in India \& SOP On Culture and Drug Susceptibility testing Procedures. Central TB Division, Directorate general of health Services, Ministry of health and Family Welfare, Nirman Bhavan, New Delhi, India.

17. Chauhan, M.M.; Mahadev, B., Balasangameshwara, V.H.1998 Manual on isolation, identification and sensitivity testing of Mycabaterium tuberculosis. Manual. National Tuberculosis Institute, Bangalore. India.

18. Mycobacterium tuberculosis culture identification test. Accuprobe manual.1993.Gen-Probe Inc., San Diego, Calif

19. Alert, H., Heydenrych, A., Mole, R., Trollig, A., Blumberg L.(2001)Evaluation of FAST plaque TB-RIF ${ }^{\mathrm{TM}}$, a rapid, manual test for the determination of rifampicin resistance from mycobacterium tuberculosis cultures. Int. J. Tuberc. Lung Dis. 5, 906-911.

20. Caniedes, L., Lee, T.S., Gilman, R.H., Sheen, P., Spellman, E., Lee, E.H., Berg, D.E., James S.M., and the tuberculosis working group in Peru. Rapid, efficient detection and drug susceptibility testing of mycobacterium tuberculosis in sputum by microscopic observation of both cultures. J. Clin. Microbiol.2000;38: 1203-1208.

21. Diaz Infantes, M.S., Ruiz-Serrano, M.J., Martinez Sancho, L., Oprtega, A., Bouz, E. Evaluation of the MB/BacT mycobacterium, detection system for susceptibility testing of Mycobacterium tuberculosis. J. Clin. Microbiol.2000;38: 1988-1989.

22. Mahadev, B.Comparison between rapid colorimetric mycobacterial isolation and susceptibility testing method and conventional method using LJ medium. Ind. J. Tuberculosis.2001;48:129-134.

23. Tenover, F.C., Crawford, J.T., Huebner, R.E., Goiter, L.J., Horsburgh Jr, C.R., Good, R.C.The resurgence of tuberculosis is your laboratory ready? J Clin Microbiol.1993;31:767-770.

24. Madison, B.B., Robinson-Dunn, George,I., Gross,W., Lipman,H., Metchock,B., Sloutsky,A., Washabaugh,G., Mazurek,G., Reddenhof,J. Multicenter evaluation of ethambutol susceptibility testing of Mycobacterium tuberculosis by agar proportion method and radiometric methods. J Clin Microbiol 2002;40(11): 3976-3979.

25. Hemrani, N. and Chitnis D.S.Prevalence of drug resistance among M.tuberculosis isolates in Indore (MP). Ind. J. Med. Microbiol.2002;20:228.

26. Malhotra, B., Pathak, S., Vyas, L., Katoch, V.M., Srivastava, K., Chauhan, D.S., Singh, D., Sharma, V.D., Das, R., Singh, H.B. Drug susceptibility profiles of Mycobacterium tuberculosis isolates at Jaipur. Ind J Med Microbiol 2002;20: 76-78.

27. Singh, S. Scaling up anti-mycobacterial drug susceptibility testing services in India:it is high time. Ind. J. Med. Microbiol.2008; 26:209-211.

28. Butt, T., Ahmad, R.N., Kazmi, S.Y., Rafi, N.Multidrug resistant tuberculosis in Northern Pakistan. J. Pak. Med. Assoc. 2004;54:469-72. 